\title{
Risk of infection and adverse outcomes among pregnant working women in selected occupational groups: A study in the Danish National Birth Cohort
}

\author{
Maria Morales-Suárez-Varela ${ }^{1,2,3^{*}}$, Linda Kaerlev ${ }^{4,5}$, Jin Liang Zhu4 ${ }^{4}$, Agustín Llopis-González ${ }^{1,2,3}$, \\ Natalia Gimeno-Clemente ${ }^{1,2,3}$, Ellen A Nohr ${ }^{4}$, Jens P Bonde ${ }^{6}$, Jorn Olsen ${ }^{6,7}$
}

\begin{abstract}
Background: Exposure to infectious pathogens is a frequent occupational hazard for women who work with patients, children, animals or animal products. The purpose of the present study is to investigate if women working in occupations where exposure to infections agents is common have a high risk of infections and adverse pregnancy outcomes.

Methods: We used data from the Danish National Birth Cohort, a population-based cohort study and studied the risk of Infection and adverse outcomes in pregnant women working with patients, with children, with food products or with animals. The regression analysis were adjusted for the following covariates: maternal age, parity, history of miscarriage, socio-occupational status, pre-pregnancy body mass index, smoking habit, alcohol consumption.
\end{abstract}

Results: Pregnant women who worked with patients or children or food products had an excess risk of sick leave during pregnancy for more than three days. Most of negative reproductive outcomes were not increased in these occupations but the prevalence of congenital anomalies (CAs) was slightly higher in children of women who worked with patients. The prevalence of small for gestational age infants was higher among women who worked with food products. There was no association between occupation infections during pregnancy and the risk of reproductive failures in the exposed groups. However, the prevalence of CAs was slightly higher among children of women who suffered some infection during pregnancy but the numbers were small.

Conclusion: Despite preventive strategies, working in specific jobs during pregnancy may impose a higher risk of infections, and working in some of these occupations may impose a slightly higher risk of CAs in their offspring. Most other reproductive failures were not increased in these occupations.

\section{Background}

An estimated, 320,000 workers worldwide die every year of infection diseases caused by virus, bacteria, and other microorganism [1]. Most pregnant women should be able to continue their work in a workplace free of these reproductive hazards [2], but for women working in close contact with children or sick people, this may be difficult to achieve $[3,4]$. Some health care workers and childcare workers are often exposed to infectious

\footnotetext{
* Correspondence: maria.m.morales@uv.es

'Unit of Public Health and Environmental Care, Department of Preventive

Medicine, University of Valencia, Valencia, Spain

Full list of author information is available at the end of the article
}

pathogens at work $[5,6]$. Furthermore, infections may be a common occupational hazard for food handlers and persons in waste management and sewage work [7].

A number of environmental factors [8], including infections [9], have been implicated in adverse pregnancy outcomes and the teratogenicity of some infections is well documented [3] such as cytomegalovirus (CMV), rubella, parvovirus B19, herpes and toxoplasmosis. Common cold is the most frequently reported maternal infection during pregnancy, but its possible association with adverse pregnancy outcomes is not well studied, and many different viruses are involved [10].

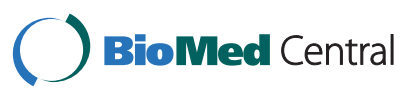

(c) 2010 Morales-Suárez-Varela et al; licensee BioMed Central Ltd. This is an Open Access article distributed under the terms of the Creative Commons Attribution License (http://creativecommons.org/licenses/by/2.0), which permits unrestricted use, distribution, and reproduction in any medium, provided the original work is properly cited. 
Infections have been reported to cause spontaneous abortion and fetal death [3,11], preterm birth [3,12], intrauterine growth restriction [3,13], and birth defects, including abnormalities of the central nervous system $[3,14]$, ophthalmologic manifestations $[3,15]$, and congenital heart defects $[3,16]$. The type of adverse effects may vary with gestational age at the time of infection $[3,16]$. Most infections, however, leave no identifiable trace of damage.

Since infections often are reported with misclassification we base our analyses on occupational titles using high risk occupations as an instrumental variable for exposure. We then check if these occupations are characterized by having a high frequency of infections. Finally we check if those who reported infections in these occupations give birth to children with more reproductive failures.

The aim of this study was to investigate if pregnancy outcomes are associated with occupations involving a potential high risk of infections.

\section{Methods}

\section{Population}

Data were obtained from the Danish National Birth Cohort (DNBC), which is a nationwide study among pregnant women and their offspring. The study is described in detail elsewhere [17]. Between March 1996 and November 2002, pregnant women across Denmark were invited to participate in the study by their general practitioner. Approximately 50\% of all general practitioners took part in the recruitment. The exclusion criteria were not having access to a telephone, not speaking Danish well enough to complete the interview, and not intending to carry the pregnancy to term at the first visit. About $60 \%$ of those invited chose to participate in the study and signed an informed consent form [18].

A total of 101,047 pregnancies were enrolled in the study, and for 90,301 of these, the women had participated in the first interview of the study that took place at approximately 16 weeks of gestation (interquartile range, 12-20). The data was obtained by computerassisted telephone interviews. Interviews were missing if the woman could not be reached at the scheduled time or at three additional contact attempts and the interview was cancelled if the woman was no longer pregnant at the time of the interview.

If the women who participated in the first telephone interview had more than one pregnancy during the study period, we included only the first pregnancy and excluded all subsequent pregnancies $(n=2,425)$. Also, we excluded women if their pregnancy was terminated by an induced abortion ( $\mathrm{n}=93)$, hydatidiform mole ( $\mathrm{n}$ $=42)$, ectopic pregnancies $(n=24)$, or if they had multiple birth with no live born infants $(n=3)$ or if the mother died during pregnancy $(n=1)$.

\section{Occupational settings}

In the first interview of the study, 83,448 mothers answered questions about their main occupation during pregnancy and three months prior to pregnancy. We excluded unemployed women $(\mathrm{n}=18,071)$.

The occupation of 65,377 women was later coded according to the Danish Version of International Standard Classification of Occupation (DISCO-88) $[19,20]$. Women were then categorised to each of the following occupational settings: A) contact with patients ( $\mathrm{n}=$ $8,699)$, B) contact with children $(\mathrm{n}=9,151), \mathrm{C})$ contact with food products $(\mathrm{n}=932)$, D) contact with animals $(\mathrm{n}=287)$, and E) the rest were classified as "unexposed workers" ( $n=46,308)$. The groups consisted of A) chemist's assistants $(n=4)$, physiotherapists $(n=583)$, dental nurses $(\mathrm{n}=444)$, laboratory technicians $(\mathrm{n}=170)$, doctors $(\mathrm{n}=566)$, auxiliary nurses $(\mathrm{n}=2,681)$ and nurses $(\mathrm{n}=4,251), B)$ child-minders $(\mathrm{n}=641)$, specialist teachers $(\mathrm{n}=274)$, teachers of common school $(\mathrm{n}=$ $2,456)$, upper secondary school teachers $(n=167)$, care assistant $(n=116)$, educationalists $(n=3,684)$ and educationalist assistants $(\mathrm{n}=1,813)$ C) kitchen assistants $(\mathrm{n}=842)$, butchers $(\mathrm{n}=90)$ and $\mathrm{D})$ skilled farm workers $(\mathrm{n}=124)$, slaughter house workers $(\mathrm{n}=163)$, and $\mathrm{E})$ all other workers $(n=46,308)$ (Figure 1$)$.

\section{Infections}

We collected self-reported information about infections during pregnancy in the second interview carried out approximately at week 30 (interquartile range, 25-32). The women were asked whether she at any point in time during pregnancy 1) had been absent due to illness for more than three days (yes/no) and the number of times she had been absent (never, one-four and more than four times), 2) if she had taken any medications for infectious diseases (yes/no), 3) if she had had an episode of fever (yes/no) and the number of episodes (never, one-two episodes, and more than two episodes), 4) whether she had had diarrhoea (yes/no) and the number of episodes (never, one-four and more than four episodes),5) if she had had cold sore or oral herpes (yes/ no), 6) if she had had cold sore or genital herpes (yes/ no), 7) if she had had cystitis (yes/no), and 8) if she had had a skin infection (yes/no).

\section{Pregnancy outcomes}

We identified pregnancy outcomes in the National Hospital Discharge Register (NHDR) and the Danish Medical Birth Register by register linkage using the unique personal identification number (civil registration number) which is assigned to all Danish residents at birth. 


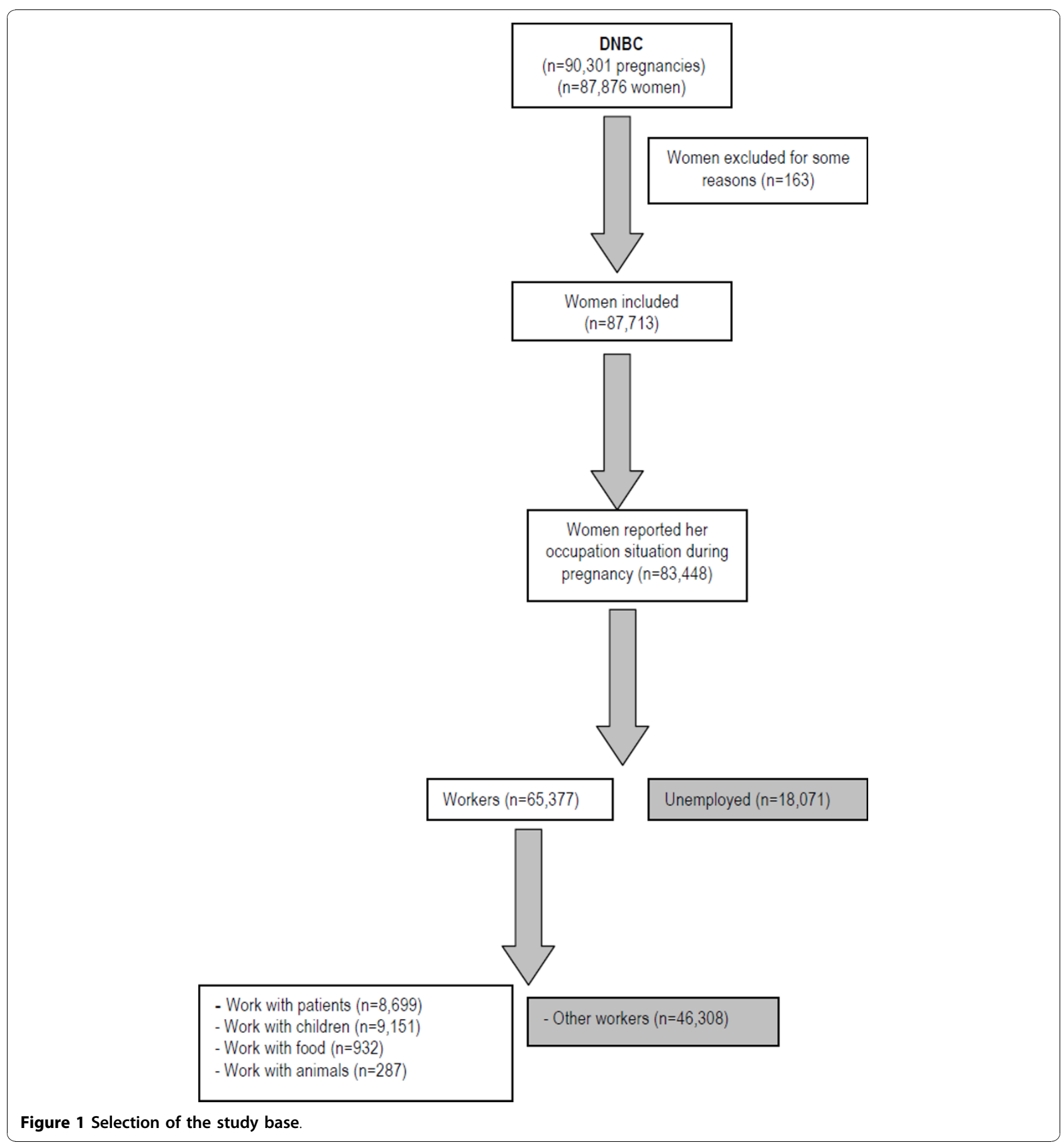

Information about gestational age also came from the National Hospital Discharge Register and was mainly based on ultrasound examination.

We studied miscarriages (late spontaneous abortions and stillbirth), sex ratio (male/female), preterm birth $(<$ 37 weeks of gestation) and very preterm birth $(<34$ weeks of gestation), small-for-gestational-age status $(<$ the $10^{\text {th }}$ percentile for the sex - and gestation-specific birth weight in the DNBC by week of gestation) and
APGAR (Appearance (skin color), Pulse (heart rate), Grimace (reflex irritability), Activity (muscle tone), and Respiration) [21]) score at five minutes of birth. We also studied congenital anomalies (CAs) according to the codes DQ00-DQ99 from the 10th Revision of International Classification of Diseases (ICD10). We used the European Surveillance of Congenital Abnormalities Classification (EUROCAT) [22] to categorize CAs as minor and major, and excluded chromosome aberrations, 
genetic syndromes and microdeletions, teratogenic syndrome. We used all CAs and major CAs diagnosed at birth or during the first year of life.

\section{Other variables}

From the first interview, we obtained information about maternal age, parity, pre-pregnancy body mass index (BMI), smoking habits and alcohol consumption during pregnancy, and a history of miscarriage (spontaneous abortions).

Mothers were classified into three categories of sociooccupational status based upon their job title: high, medium or low. Women with a higher level of education (four years beyond high school) or in management positions were classified as "high socio-occupational status". The "medium" category included skilled workers and women with medium-ranged training/level of education, while the "low" category included workers with no formal skilled training [23].

\section{Statistical Analysis}

Odds ratios (ORs) and 95\% confidence intervals (95\%CI) were used to compare risks of infections in women in occupational settings more prone to infectious exposure (A-D above) with the risks in other women (E). Also, in each working group, we compare risks of adverse pregnancy outcomes in women who suffered some infection with the risks in women who did not suffer any infection during pregnancy.

Multiple logistic regression models were applied to adjust for the following potential confounders: Maternal age: $<30,30-34$, or $>35$ years of age; parity: nulliparous women (0) or multiparous women $(1+)$; history of miscarriage: yes or no; pre-pregnancy BMI: underweight (< 18.5), normal weight (18.5-24.9), overweight (25.0-29.9), and obese $(30.0+)$; smoking habit during pregnancy: 0 , $1-9,10-19$, or $>20$ cig./day; alcohol consumption during pregnancy: $0,0.1-19.9,20.0-39.9$ or $>40.0$ gr./day and socio-occupational status of the mother: high, medium or low.

All the analyses were performed using the SPSS software (version 17.0; SPSS Inc. Chicago III).

The Danish Data Protection Agency approved the study.

\section{Results}

Maternal characteristics are summarized in Table 1. Mothers who worked with patients reported a higher frequency of previous miscarriages and use of fertility treatment prior to the present pregnancy. Compared to the other occupational groups, mothers who worked with food products or with animals were younger and of lower socio-occupational status. They had a higher prepregnancy BMI, were more often smokers, smoked more cigarettes per day, but reported lower alcohol consumption. They reported the lowest history of miscarriages of all groups.

Table 2 shows occupational groups and symptoms of infections. When we adjusted for additional variables (maternal age, parity, history of miscarriage, sociooccupational status, pre-pregnancy BMI, smoking habit and alcohol consumption) we found that pregnant women who work with patients had a higher risk of sick leave for more than three days, medically treated infections, oral herpes and cystitis. Women who worked with children had an increased risk of sick leave for more than three days, an episode of fever, and skin infections during pregnancy. Finally, pregnant women who worked with food products had an excess risk of sick leave for more than three days.

Table 3 shows no difference between the different occupational groups for the risk of miscarriages, sex ratio, preterm and very preterm birth, and APGAR $<7$ at five minutes, but the prevalence of CAs was higher among children of women who worked with patients (ORa 1.09, 95\% CI: 1.00-1.18 for all live births and ORa 1.10, 95\% CI: $1.00-1.20$ for all live born singletons), as well as major CAs (ORa 1.11, 95\% CI 1.00-1.22). The prevalence of small for gestational age infants was higher among women who worked with food products (ORa 1.33, 95\% CI: 1.07-1.59).

In table 4 we analyse if those who reported infections in these "exposed occupations" had children with more reproductive failures. We observe no association between reportly of some infection during pregnancy and the risk of reproductive failures in the exposed groups. However, in women who work with animals, the prevalence of CAs was slightly higher among children of women who suffered one or more infections (ORa 2.93, 95\% CI: $1.24-4.62$ for all live births and ORa 2.80, 95\% CI: 1.23-4.37 for all live born singletons), as well as major CAs (ORa 2.80, 95\% CI: 1.23-4.37).

\section{Discussion}

Absence from work for more than three days during pregnancy because of illness was more frequent in women who worked with patients, children or food products compared with other women. Women who worked with patients also reported more infectious diseases treated with medication, oral herpes and cystitis episodes. Women who worked with patients also had more frequent fever episodes and skin infections. On the other hand, woman who worked with patients had a higher risk of CAs and woman who worked with food products had a higher risk of having children who were small for gestational age. In the women who work with animals the prevalence of CAs was higher among children of women who suffered some infection than 
Table 1 Characteristics of the study population

\begin{tabular}{|c|c|c|c|c|c|c|c|c|c|c|c|c|}
\hline & \multicolumn{2}{|c|}{$\begin{array}{l}\text { Work with } \\
\text { patients }^{1} \\
(\mathrm{~N}=8699)\end{array}$} & \multicolumn{2}{|c|}{$\begin{array}{l}\text { Work with } \\
\text { children } \\
(\mathrm{N}=9151)\end{array}$} & \multicolumn{2}{|c|}{$\begin{array}{l}\text { Work with } \\
\text { food }^{3} \\
(\mathrm{~N}=932)\end{array}$} & \multicolumn{2}{|c|}{$\begin{array}{c}\text { Work } \\
\text { with } \\
\text { animals } \\
(\mathrm{N}=287)\end{array}$} & \multicolumn{2}{|c|}{$\begin{array}{c}\text { Total exposed } \\
\text { workers } \\
(\mathrm{N}=19069)\end{array}$} & \multicolumn{2}{|c|}{$\begin{array}{c}\text { Other } \\
\text { workers } \\
(\mathrm{N}=46308)\end{array}$} \\
\hline & $\mathrm{N}$ & $\%$ & $\mathrm{~N}$ & $\%$ & $\mathrm{~N}$ & $\%$ & $\mathrm{~N}$ & $\%$ & $\mathrm{~N}$ & $\%$ & $\mathrm{~N}$ & $\%$ \\
\hline \multicolumn{13}{|l|}{ Maternal age, years } \\
\hline$<30$ & 4166 & 48.7 & 4313 & 48.0 & 569 & 62.3 & 168 & 59.2 & 9216 & 49.2 & 19724 & 43.3 \\
\hline $30-34$ & 3089 & 36.1 & 3340 & 37.2 & 246 & 26.9 & 84 & 29.6 & 6759 & 36.1 & 18425 & 40.5 \\
\hline $35+$ & 1294 & 15.1 & 1335 & 14.9 & 99 & 10.8 & 32 & 11.3 & 2760 & 14.7 & 7358 & 16.2 \\
\hline Total & 8549 & 100.0 & 8988 & 100.0 & 914 & 100.0 & 284 & 100.0 & 18735 & 100.0 & 45507 & 100.0 \\
\hline \multicolumn{13}{|l|}{ Parity } \\
\hline 0 & 3354 & 38.6 & 3465 & 37.9 & 332 & 35.7 & 98 & 34.1 & 7249 & 38.0 & 17701 & 38.2 \\
\hline $1+$ & 5342 & 61.4 & 5684 & 62.1 & 599 & 64.3 & 189 & 65.9 & 11814 & 62.0 & 28584 & 61.8 \\
\hline Total & 8696 & 100.0 & 9149 & 100.0 & 931 & 100.0 & 287 & 100.0 & 19063 & 100.0 & 46285 & 100.0 \\
\hline \multicolumn{13}{|c|}{ Pre-pregnancy body mass index, $\mathrm{kg} / \mathrm{m}^{2}$} \\
\hline Underweight $(<18.5)$ & 75 & 0.9 & 61 & 0.7 & 9 & 1.1 & 9 & 0.1 & 145 & 0.8 & 530 & 1.3 \\
\hline Normal weight (18.5-24.9) & 5183 & 64.7 & 4802 & 58.6 & 376 & 44.8 & 125 & 48.8 & 10486 & 60.6 & 26081 & 62.1 \\
\hline Overweight (25.0-29.9) & 1949 & 24.3 & 2272 & 27.7 & 279 & 33.2 & 78 & 30.5 & 4578 & 26.5 & 10997 & 26.2 \\
\hline Obese/very obese (30.0+) & 800 & 10.0 & 1064 & 13.0 & 176 & 21.0 & 53 & 20.7 & 2093 & 12.1 & 4381 & 10.4 \\
\hline Total & 8007 & 100.0 & 8199 & 100.0 & 840 & 100.0 & 256 & 100.0 & 17302 & 100.0 & 41989 & 100.0 \\
\hline \multicolumn{13}{|l|}{ Smoking habit } \\
\hline No & 6608 & 76.0 & 6779 & 76.0 & 619 & 66.5 & 182 & 63.4 & 14188 & 74.4 & 34709 & 75.0 \\
\hline Yes & 2086 & 24.0 & 2086 & 24.0 & 312 & 33.5 & 105 & 36.6 & 4872 & 25.6 & 11586 & 25.0 \\
\hline Total & 8694 & 100.0 & 8694 & 100.0 & 931 & 100.0 & 287 & 100.0 & 19060 & 100.0 & 46295 & 100.0 \\
\hline \multicolumn{13}{|l|}{ Smoking habit, cig./day ${ }^{*}$} \\
\hline 0 & 6627 & 76.2 & 6807 & 74.4 & 623 & 66.9 & 183 & 63.8 & 14240 & 74.7 & 34806 & 75.2 \\
\hline $1-9$ & 1193 & 13.7 & 1364 & 14.9 & 162 & 17.4 & 40 & 13.9 & 2759 & 14.5 & 6483 & 14.0 \\
\hline $10-19$ & 758 & 8.7 & 850 & 9.3 & 123 & 13.2 & 55 & 19.2 & 1786 & 9.4 & 4241 & 9.2 \\
\hline $20+$ & 116 & 1.3 & 127 & 1.4 & 23 & 2.5 & 9 & 3.1 & 275 & 1.4 & 766 & 1.7 \\
\hline Total & 8694 & 100.0 & 9148 & 100.0 & 931 & 100.0 & 287 & 100.0 & 19060 & 100.0 & 46296 & 100.0 \\
\hline \multicolumn{13}{|l|}{ Alcohol consumption, gr./week } \\
\hline 0 & 6278 & 72.2 & 6788 & 74.2 & 748 & 80.3 & 232 & 80.8 & 14046 & 73.7 & 32222 & 69.6 \\
\hline $0.1-19.9$ & 149 & 1.7 & 157 & 1.7 & 5 & 0.5 & 3 & 1.0 & 314 & 1.6 & 744 & 1.6 \\
\hline 20-39.9 & 1470 & 16.9 & 1412 & 15.4 & 124 & 13.3 & 32 & 11.1 & 3038 & 15.9 & 8473 & 18.3 \\
\hline $40+$ & 802 & 9.2 & 794 & 8.7 & 55 & 5.9 & 20 & 7.0 & 1671 & 8.8 & 4869 & 10.5 \\
\hline Total & 8699 & 100.0 & 9151 & 100.0 & 932 & 100.0 & 287 & 100.0 & 19069 & 100.0 & 46308 & 100.0 \\
\hline \multicolumn{13}{|l|}{ History of miscarriage $^{\dagger}$} \\
\hline No & 7142 & 82.2 & 7592 & 83.0 & 775 & 83.2 & 246 & 85.7 & 15755 & 82.7 & 38282 & 82.7 \\
\hline Yes & 1550 & 17.8 & 1555 & 17.0 & 156 & 16.8 & 41 & 14.3 & 3302 & 17.3 & 7984 & 17.3 \\
\hline Total & 8692 & 100.0 & 9147 & 100.0 & 931 & 100.0 & 287 & 100.0 & 19057 & 100.0 & 46266 & 100.0 \\
\hline \multicolumn{13}{|l|}{ Infertility treatment } \\
\hline No & 8045 & 92.5 & 8565 & 93.6 & 876 & 94.0 & 277 & 96.5 & 17763 & 93.2 & 42780 & 92.4 \\
\hline Yes & 649 & 7.5 & 586 & 6.4 & 56 & 6.0 & 10 & 3.5 & 1301 & 6.8 & 3505 & 7.6 \\
\hline Total & 8694 & 100.0 & 9151 & 100.0 & 932 & 100.0 & 287 & 100.0 & 19064 & 100.0 & 46285 & 100.0 \\
\hline \multicolumn{13}{|c|}{ Work at the time of the $1^{\text {st }}$ interview* } \\
\hline No & 298 & 3.4 & 428 & 4.7 & 82 & 8.8 & 32 & 11.1 & 840 & 4.4 & 171 & 0.4 \\
\hline Yes & 8400 & 96.6 & 8722 & 95.3 & 849 & 91.2 & 255 & 88.9 & 18226 & 95.6 & 41039 & 99.6 \\
\hline Total & 8698 & 100.0 & 9150 & 100.0 & 931 & 100.0 & 287 & 100.0 & 19066 & 100.0 & 41210 & 100.0 \\
\hline \multicolumn{13}{|l|}{ Socio-occupational status } \\
\hline High & 6484 & 74.5 & 7386 & 80.7 & 213 & 22.9 & 49 & 17.1 & 14132 & 74.1 & 30294 & 65.5 \\
\hline Medium & 2210 & 25.4 & 1762 & 19.3 & 719 & 77.1 & 168 & 58.5 & 4859 & 25.5 & 14564 & 31.5 \\
\hline
\end{tabular}


Table 1 Characteristics of the study population (Continued)

\begin{tabular}{|c|c|c|c|c|c|c|c|c|c|c|c|c|}
\hline Low & 4 & 0.1 & 2 & 0.1 & 0 & 0 & 70 & 24.4 & 76 & 0.4 & 1421 & 3.1 \\
\hline Total & 8698 & 100.0 & 9150 & 100.0 & 932 & 100.0 & 287 & 100.0 & 19067 & 100.0 & 46279 & 100.0 \\
\hline
\end{tabular}

${ }^{1}$ Health care and laboratory workers in hospitals.

${ }^{2}$ Day care centres workers and school teacher.

${ }^{3}$ Kitchen assistant, waitress and butcher.

${ }^{4}$ Skiled farm worker, slaughter house worker.

${ }^{¥} 1$ cigarette $=1$ cigarette equivalent, 1 cherrot $=2$ cigarette equivalent, 1 cigar $=2$ cigarette equivalent, 1 pipe $=1.5$ cigarette equivalent.

${ }^{\dagger}$ Among singletons.

*First tree month of the pregnant.

among children of women who did not suffer any infection during pregnancy. In all the other working groups there was no association between the suffering of some infection during pregnancy and the risk of any other adverse pregnancy outcomes.

The higher risk of CAs we found in woman who worked with patients was previously been reported using data from the DNBC [24]. On the other hand, it is known that many people employed in the health care sector are exposed to a wide variety of contagious diseases and the diversity of potentially infectious agents continues to increase [25]. Health professionals however had a higher risk of cytomegalovirus as well as other herpes virus. Some studies show that maternal infected leukocytes cross the placental tissue and amniotic cells, which are swallowed by the foetus and may cause congenital infection [26] and harm the foetal central nervous system, eyes and liver [27]. Cystitis has been reported to be the most common type of infections in pregnant woman [28] not least in the group working with patients. This condition may be responsible for some of the adverse pregnancy outcomes among health care workers [29].

Other studies have shown that day care centre workers and school teachers in close contact with children are more often exposed to infections in the workplace [30]. Infectious agents like Cytomegalovirus and Parvovirus B19 have been shown to frequently affect women who work with children [31], but we did not find an excess risk of any adverse pregnancy outcome in this group.

In the group of women who work with animals, we did not find any indication of a high exposure to infections that affect humans than in the group of other workers. However, we found a higher prevalence of CAs among children of women who reported some infections during pregnancy.

In this large population-based cohort study, we collected information prospectively, had almost complete follow up on more than 83,000 pregnancies, and could carry our extensive confounder adjustment. The impact of the participation rate on the Danish National Birth Cohort was examined in a study among 49,751 women from the source population, including 15,373 participants in the cohort study. This study concludes that its results have been not biased by nonparticipation. These results are reassuring for studies based on the Danish cohort as the present study [18]. However, our study also has weaknesses. Although we adjusted for a number of potential confounders, confounding of the results by other factors related to working with patients or with children is possible (i.e., ionising radiation [32], magnetic fields [33], chemical substances [34], shiftwork [35] or physically demanding work [36]). A high level of sick leave during pregnancy has been reported in jobs with a high risk of infecting others [37]. This may, however, just reflect a lower threshold for sick leaves if women work with other people.

The ad hoc classification of the job titles that we used in this study was not based on a formerly used Job exposure matrix, we used self-reported information about infections, and confounding by other diseases associated may also have had effect on development of CA. We acknowledge that some of the adjusted estimates for rare outcomes were based on small numbers, but the imprecision was reflected in the width of the corresponding 95\% confidence intervals. Furthermore, some of the results may be due to chance based on multiple comparisons. Finally, we investigate the miscarriage only after the first interview, which was around 16 weeks of gestation. Exposure leading to early miscarriage or abortion thus cannot be identified in this study and the actual rate of adverse pregnancy outcomes such as CAs may be underestimated if the women had an induced abortion following a positive prenatal test of CA's.

The purpose of the present study was to investigate I) whether fever or infections during pregnancy were more common in certain job groups, 2) whether the pregnant women having these jobs that entailed a high risk of infection were at increased risk pregnancy outcomes, and 3) whether in these occupational groups were association between the suffering of some infection during pregnancy and the risk of pregnancy outcomes. We did not find any strong indication of excess risk in these occupations. Nor did we find that woman in these 
Table 2 Crude and adjusted odds ratios (OR) for infections, symptoms and absence from work due to sickness according to occupation

\begin{tabular}{|c|c|c|c|c|c|c|c|c|c|c|c|c|c|c|c|c|c|c|}
\hline \multirow[t]{2}{*}{$\begin{array}{l}\text { During the } \\
\text { pregnancy }\end{array}$} & \multicolumn{2}{|c|}{$\begin{array}{c}\text { Other } \\
\text { workers } \\
(\mathrm{N}= \\
46308)\end{array}$} & \multicolumn{4}{|c|}{$\begin{array}{l}\text { Work with patients }{ }^{1} \\
\quad(\mathrm{~N}=8699)\end{array}$} & \multicolumn{4}{|c|}{$\begin{array}{l}\text { Work with children }{ }^{2} \\
\quad(\mathrm{~N}=9151)\end{array}$} & \multicolumn{4}{|c|}{$\begin{array}{l}\text { Work with food } \\
\quad(N=932)\end{array}$} & \multicolumn{4}{|c|}{$\begin{array}{l}\text { Work with } \\
\text { animals } \\
(\mathrm{N}=287)\end{array}$} \\
\hline & $N$ & $\%$ & $N$ & $\%$ & $\begin{array}{c}\text { ORc } \\
(95 \% \\
\mathrm{Cl}) \\
\end{array}$ & $\begin{array}{c}\text { ORa } \\
(95 \% \\
\mathrm{Cl}) \\
\end{array}$ & $N$ & $\%$ & $\begin{array}{c}\text { ORc } \\
(95 \% \\
\mathrm{Cl}) \\
\end{array}$ & $\begin{array}{c}\text { ORa } \\
(95 \% \\
\mathrm{Cl}) \\
\end{array}$ & $\mathrm{N}$ & $\%$ & $\begin{array}{l}\text { ORc } \\
(95 \% \\
\mathrm{Cl}) \\
\end{array}$ & $\begin{array}{c}\text { ORa } \\
(95 \% \\
\mathrm{Cl}) \\
\end{array}$ & $\mathbf{N}$ & $\%$ & $\begin{array}{c}\text { ORc } \\
(95 \% \\
\text { Cl) } \\
\end{array}$ & $\begin{array}{l}\text { ORa } \\
(95 \% \\
\mathrm{Cl}) \\
\end{array}$ \\
\hline $\begin{array}{l}\text { Sickness for more } \\
\text { leave than } 3 \text { days }\end{array}$ & 9160 & 27.3 & 2347 & 38.7 & $\begin{array}{c}1.68 \\
(1.58- \\
1.78)\end{array}$ & $\begin{array}{c}1.80 \\
(1.70- \\
1.90)\end{array}$ & 2273 & 34.4 & $\begin{array}{c}1.39 \\
(1.33- \\
1.47)\end{array}$ & $\begin{array}{c}1.47 \\
(1.38- \\
1.56)\end{array}$ & 218 & 37.1 & $\begin{array}{r}1.58 \\
(1.34- \\
1.82)\end{array}$ & $\begin{array}{c}1.43 \\
(1.20- \\
1.66)\end{array}$ & 58 & 40.3 & $\begin{array}{l}1.80 \\
(1.29- \\
2.31)\end{array}$ & $\begin{array}{r}1.10 \\
(0.80- \\
1.40)\end{array}$ \\
\hline \multicolumn{19}{|l|}{$\begin{array}{l}\text { Episodes of sickness } \\
\text { for more leave than } 3 \\
\text { days }\end{array}$} \\
\hline $1-4$ & 8808 & 26.3 & 2239 & 37.0 & $\begin{array}{l}1.67 \\
(1.57- \\
1.77)\end{array}$ & $\begin{array}{r}1.79 \\
(1.68- \\
1.90)\end{array}$ & 2165 & 32.8 & $\begin{array}{c}1.38 \\
(1.30- \\
1.46)\end{array}$ & $\begin{array}{r}1.45 \\
(1.35- \\
1.55)\end{array}$ & 207 & 35.3 & $\begin{array}{r}1.55 \\
(1.30- \\
1.85)\end{array}$ & $\begin{array}{r}1.40 \\
(1.17- \\
1.63)\end{array}$ & 58 & 40.3 & $\begin{array}{l}1.86 \\
(1.34- \\
2.38)\end{array}$ & $\begin{array}{r}1.14 \\
(0.80- \\
1.48)\end{array}$ \\
\hline $5+$ & 301 & 0.9 & 96 & 1.6 & $\begin{array}{l}2.09 \\
(1.66- \\
2.52)\end{array}$ & $\begin{array}{l}2.19 \\
(1.72- \\
2.80)\end{array}$ & 95 & 1.4 & $\begin{array}{l}1.77 \\
(1.40- \\
2.24)\end{array}$ & $\begin{array}{l}1.86 \\
(1.45- \\
2.27)\end{array}$ & 11 & 1.9 & $\begin{array}{c}2.41 \\
(1.31- \\
3.51)\end{array}$ & $\begin{array}{c}2.22 \\
(1.19- \\
3.25)\end{array}$ & 0 & - & - & - \\
\hline $\begin{array}{l}\text { Any infection with } \\
\text { medication }\end{array}$ & 7379 & 17.3 & 1510 & 18.8 & $\begin{array}{r}1.10 \\
(1.04- \\
1.16)\end{array}$ & $\begin{array}{c}1.09 \\
(1.02- \\
1.16)\end{array}$ & 1563 & 18.6 & $\begin{array}{c}1.09 \\
(1.03- \\
1.15)\end{array}$ & $\begin{array}{l}1.05 \\
(0.98- \\
1.12)\end{array}$ & 139 & 16.4 & $\begin{array}{c}0.93 \\
(0.78- \\
1.08)\end{array}$ & $\begin{array}{c}0.92 \\
(0.76- \\
1.08)\end{array}$ & 46 & 18.2 & $\begin{array}{l}1.06 \\
(0.77- \\
1.35)\end{array}$ & $\begin{array}{c}1.04 \\
(0.74- \\
1.44)\end{array}$ \\
\hline Fever & 9412 & 22.2 & 1904 & 23.8 & $\begin{array}{l}1.34 \\
(0.97- \\
1.71)\end{array}$ & $\begin{array}{l}1.05 \\
(0.99- \\
1.11)\end{array}$ & 2086 & 24.9 & $\begin{array}{c}1.47 \\
(1.06- \\
1.88)\end{array}$ & $\begin{array}{r}1.10 \\
(1.04- \\
1.16)\end{array}$ & 164 & 19.3 & $\begin{array}{r}1.56 \\
(1.12- \\
2.00)\end{array}$ & $\begin{array}{c}0.90 \\
(0.74- \\
1.06)\end{array}$ & 44 & 17.5 & $\begin{array}{c}1.12 \\
(0.78- \\
1.46)\end{array}$ & $\begin{array}{c}0.82 \\
(0.59- \\
1.05)\end{array}$ \\
\hline \multicolumn{19}{|l|}{ Episodes of fever } \\
\hline $1-2$ & 5124 & 12.1 & 1006 & 12.6 & $\begin{array}{c}1.04 \\
(0.96- \\
1.12)\end{array}$ & $\begin{array}{c}1.01 \\
(0.92- \\
1.10)\end{array}$ & 1051 & 12.6 & $\begin{array}{c}1.04 \\
(0.96- \\
1.12)\end{array}$ & $\begin{array}{c}0.99 \\
(0.91- \\
1.07)\end{array}$ & 87 & 10.3 & $\begin{array}{c}0.83 \\
(0.66- \\
1.00)\end{array}$ & $\begin{array}{c}0.85 \\
(0.68- \\
1.05)\end{array}$ & 27 & 10.8 & $\begin{array}{c}0.87 \\
(0.58- \\
1.16)\end{array}$ & $\begin{array}{c}0.91 \\
(0.60- \\
1.22)\end{array}$ \\
\hline $3+$ & 121 & 0.3 & 18 & 0.2 & $\begin{array}{c}0.80 \\
(0.48- \\
1.12)\end{array}$ & $\begin{array}{c}1.02 \\
(0.94- \\
1.10)\end{array}$ & 18 & 0.2 & $\begin{array}{c}0.76 \\
(0.46- \\
1.06)\end{array}$ & $\begin{array}{c}0.99 \\
(0.91- \\
1.07)\end{array}$ & 3 & 0.4 & $\begin{array}{c}1.21 \\
(0.40- \\
2.02)\end{array}$ & $\begin{array}{c}0.85 \\
(0.68- \\
1.02)\end{array}$ & 0 & - & - & - \\
\hline Diarrhoea & 7140 & 16.8 & 1237 & 15.4 & $\begin{array}{c}0.90 \\
(0.84- \\
0.96)\end{array}$ & $\begin{array}{c}0.90 \\
(0.86- \\
0.94)\end{array}$ & 1362 & 16.2 & $\begin{array}{c}0.96 \\
(0.90- \\
1.02)\end{array}$ & $\begin{array}{c}0.91 \\
(0.85- \\
0.97)\end{array}$ & 124 & 14.6 & $\begin{array}{c}0.85 \\
(0.70- \\
1.00)\end{array}$ & $\begin{array}{c}0.85 \\
(0.70- \\
1.00)\end{array}$ & 41 & 16.2 & $\begin{array}{c}0.95 \\
(0.68- \\
1.22)\end{array}$ & $\begin{array}{c}0.86 \\
(0.60- \\
1.12)\end{array}$ \\
\hline \multicolumn{19}{|l|}{ Episodes of diarrhoea } \\
\hline $1-4$ & 2284 & 5.4 & 478 & 5.9 & $\begin{array}{c}1.12 \\
(1.00- \\
1.24)\end{array}$ & $\begin{array}{c}1.13 \\
(0.99- \\
1.27)\end{array}$ & 480 & 5.7 & $\begin{array}{c}1.07 \\
(0.96- \\
1.18)\end{array}$ & $\begin{array}{c}1.09 \\
(0.97- \\
1.21)\end{array}$ & 47 & 5.5 & $\begin{array}{c}1.04 \\
(0.77- \\
1.31)\end{array}$ & $\begin{array}{c}0.88 \\
(0.65- \\
1.11)\end{array}$ & 21 & 8.3 & $\begin{array}{c}1.61 \\
(1.04- \\
2.18)\end{array}$ & $\begin{array}{c}1.00 \\
(0.61- \\
1.39)\end{array}$ \\
\hline $5+$ & 1214 & 2.8 & 243 & 3.0 & $\begin{array}{c}1.07 \\
(0.93- \\
1.21)\end{array}$ & $\begin{array}{c}1.09 \\
(0.94- \\
1.24)\end{array}$ & 247 & 2.9 & $\begin{array}{c}1.03 \\
(0.87- \\
1.19)\end{array}$ & $\begin{array}{c}1.01 \\
(0.85- \\
1.17)\end{array}$ & 31 & 3.6 & $\begin{array}{r}1.29 \\
(0.90- \\
1.68)\end{array}$ & $\begin{array}{c}1.26 \\
(0.87- \\
1.65)\end{array}$ & 10 & 4.0 & $\begin{array}{l}1.45 \\
(0.77- \\
2.13)\end{array}$ & $\begin{array}{r}1.04 \\
(0.52- \\
1.56)\end{array}$ \\
\hline $\begin{array}{l}\text { Cold sore or herpes of } \\
\text { lip }\end{array}$ & 5184 & 12.2 & 1116 & 13.9 & $\begin{array}{c}1.16 \\
(1.09- \\
1.23)\end{array}$ & $\begin{array}{c}1.19 \\
(1.10- \\
1.28)\end{array}$ & 1069 & 12.7 & $\begin{array}{c}1.05 \\
(0.98- \\
1.12)\end{array}$ & $\begin{array}{c}1.05 \\
(0.97- \\
1.13)\end{array}$ & 116 & 13.6 & $\begin{array}{c}1.14 \\
(0.93- \\
1.35)\end{array}$ & $\begin{array}{c}1.15 \\
(0.93- \\
1.37)\end{array}$ & 32 & 12.6 & $\begin{array}{c}1.05 \\
(0.72- \\
1.38)\end{array}$ & $\begin{array}{c}0.98 \\
(0.66- \\
1.30)\end{array}$ \\
\hline $\begin{array}{l}\text { Cold sore or herpes of } \\
\text { genital }\end{array}$ & 659 & 1.5 & 109 & 1.4 & $\begin{array}{c}0.88 \\
(0.71- \\
1.05)\end{array}$ & $\begin{array}{c}0.84 \\
(0.63- \\
1.05)\end{array}$ & 140 & 1.7 & $\begin{array}{c}1.08 \\
(0.87- \\
1.29)\end{array}$ & $\begin{array}{c}1.05 \\
(0.82- \\
1.28)\end{array}$ & 13 & 1.5 & $\begin{array}{c}0.99 \\
(0.57- \\
1.41)\end{array}$ & $\begin{array}{c}1.33 \\
(0.74- \\
1.92)\end{array}$ & 2 & 0.8 & $\begin{array}{c}0.50 \\
(0.01- \\
1.00)\end{array}$ & $\begin{array}{c}0.82 \\
(0.20- \\
1.44)\end{array}$ \\
\hline Cystitis & 4555 & 10.7 & 977 & 12.2 & $\begin{array}{c}1.16 \\
(1.08- \\
1.24)\end{array}$ & $\begin{array}{c}1.16 \\
(1.10- \\
1.26)\end{array}$ & 903 & 10.8 & $\begin{array}{c}1.01 \\
(0.92- \\
1.10)\end{array}$ & $\begin{array}{c}1.00 \\
(0.90- \\
1.10)\end{array}$ & 85 & 10.0 & $\begin{array}{c}0.92 \\
(0.73- \\
1.11)\end{array}$ & $\begin{array}{c}0.78 \\
(0.46- \\
1.10)\end{array}$ & 23 & 9.1 & $\begin{array}{c}0.83 \\
(0.54- \\
1.12)\end{array}$ & $\begin{array}{c}0.73 \\
(0.31- \\
1.15)\end{array}$ \\
\hline Skin infection & 293 & 0.7 & 56 & 0.7 & $\begin{array}{c}1.01 \\
(0.67- \\
1.35)\end{array}$ & $\begin{array}{c}0.99 \\
(0.65- \\
1.33)\end{array}$ & 85 & 1.0 & $\begin{array}{c}1.47 \\
(1.15- \\
1.79)\end{array}$ & $\begin{array}{c}1.50 \\
(1.16- \\
1.84)\end{array}$ & 6 & 0.7 & $\begin{array}{c}1.02 \\
(0.45- \\
1.59)\end{array}$ & $\begin{array}{c}1.10 \\
(0.48- \\
1.72)\end{array}$ & 1 & 0.4 & $\begin{array}{c}0.60 \\
(0.08- \\
1.12)\end{array}$ & $\begin{array}{c}0.56 \\
(0.08- \\
1.04)\end{array}$ \\
\hline
\end{tabular}

\footnotetext{
${ }^{1}$ chemist's assistants, physiotherapists, dental nurses, laboratory technicians, doctors, auxiliary nurses and nurses.

${ }^{2}$ child-minders, specialist teachers, teachers of common school, upper secondary school teachers, care assistant, educationalists and educationalist assistants.

${ }^{3}$ Kitchen assistant, waitress and butcher.

${ }^{4}$ Skiled farm worker, slaughter house worker.

ORc, crude Odds Ratio.

ORa' adjusted Odds Ratio for maternal age, parity, history of miscarriage, socio-occupational status, pre-pregnancy body mass index, smoking habit and alcohol consumption.
} 
Table 3 Crude and adjusted odds ratios (OR) for reproductive failures according to occupation

\begin{tabular}{|c|c|c|c|c|c|c|c|c|c|c|c|c|c|c|c|c|c|c|c|c|c|c|}
\hline & \multicolumn{3}{|c|}{$\begin{array}{c}\text { Other } \\
\text { workers } \\
(\mathrm{N}=46308)\end{array}$} & \multicolumn{3}{|c|}{$\begin{array}{l}\text { Work with patients }{ }^{1} \\
\quad(\mathrm{~N}=8699)\end{array}$} & \multicolumn{4}{|c|}{$\begin{array}{l}\text { Work with children }{ }^{2} \\
\quad(\mathrm{~N}=9151)\end{array}$} & \multicolumn{4}{|c|}{$\begin{array}{l}\text { Work with food }{ }^{3} \\
\quad(\mathrm{~N}=932)\end{array}$} & \multicolumn{4}{|c|}{$\begin{array}{l}\text { Work with animals }{ }^{4} \\
\quad(\mathrm{~N}=287)\end{array}$} & \multicolumn{4}{|c|}{$\begin{array}{l}\text { Total exposed } \\
(\mathrm{N}=19069)\end{array}$} \\
\hline & $N$ & $\%$ & $N$ & $\%$ & $\begin{array}{c}\text { ORc } \\
\text { (IC 95\%) }\end{array}$ & $\begin{array}{c}\text { ORa } \\
\text { (IC 95\%) }\end{array}$ & $\mathbf{N}$ & $\%$ & $\begin{array}{c}\text { ORc } \\
\text { (IC 95\%) }\end{array}$ & $\begin{array}{c}\text { ORa } \\
\text { (IC 95\%) }\end{array}$ & $\mathrm{N}$ & $\%$ & $\begin{array}{c}\text { ORc } \\
\text { (IC 95\%) }\end{array}$ & $\begin{array}{c}\text { ORa } \\
\text { (IC 95\%) }\end{array}$ & $\mathrm{N}$ & $\%$ & $\begin{array}{c}\text { ORc } \\
\text { (IC 95\%) }\end{array}$ & $\begin{array}{c}\text { ORa } \\
\text { (IC 95\%) }\end{array}$ & $N$ & $\%$ & $\begin{array}{c}\text { ORc } \\
\text { (IC 95\%) }\end{array}$ & $\begin{array}{c}\text { ORa } \\
\text { (IC 95\%) }\end{array}$ \\
\hline Miscarriage & 427 & 0.9 & 86 & 1.0 & $\begin{array}{c}1.07 \\
(0.85-1.29)\end{array}$ & $\begin{array}{c}1.25 \\
(0.79-1.71)\end{array}$ & 87 & 1.0 & $\begin{array}{c}1.03 \\
(0.81-1.15)\end{array}$ & $\begin{array}{c}0.93 \\
(0.57-1.25)\end{array}$ & 11 & 1.2 & $\begin{array}{c}1.28 \\
(0.70-1.86)\end{array}$ & $\begin{array}{c}1.15 \\
(0.36-1.94)\end{array}$ & 1 & 0.3 & $\begin{array}{c}0.37 \\
(0.01-1.00)\end{array}$ & - & 185 & 1.0 & $\begin{array}{c}1.05 \\
(0.90-1.20)\end{array}$ & $\begin{array}{c}1.04 \\
(0.74-1.34)\end{array}$ \\
\hline $\begin{array}{l}\text { ÆMultiple } \\
\text { births }\end{array}$ & 1050 & 2.3 & 210 & 2.5 & $\begin{array}{c}1.07 \\
(0.92-1.22)\end{array}$ & $\begin{array}{c}1.07 \\
(0.92-1.22)\end{array}$ & 170 & 1.9 & $\begin{array}{c}0.82 \\
(0.69-0.95)\end{array}$ & $\begin{array}{c}0.82 \\
(0.69-0.95)\end{array}$ & 16 & 1.7 & $\begin{array}{c}0.75 \\
(0.46-1.04)\end{array}$ & $\begin{array}{c}0.76 \\
(0.45-1.07)\end{array}$ & 2 & 0.7 & $\begin{array}{c}0.30 \\
(0.08-1.20)\end{array}$ & $\begin{array}{c}0.30 \\
(0.07-1.20)\end{array}$ & 398 & 2.1 & $\begin{array}{c}0.92 \\
(0.82-1.02)\end{array}$ & $\begin{array}{c}0.92 \\
(0.81-1.03)\end{array}$ \\
\hline $\begin{array}{l}{ }^{\dagger} \text { Male } \\
\text { infant }\end{array}$ & 22740 & & 51.2 & & 4298 & 51.5 & & 1.01 & $(0.97-1.05)$ & $\begin{array}{c}1.01 \\
(0.97-1.05)\end{array}$ & & & 4517 & 51.2 & & 1.00 & $(0.96-1.04)$ & $\begin{array}{c}1.01 \\
(0.95-1.07)\end{array}$ & 441 & 49.1 & $\begin{array}{c}0.92 \\
(0.81-1.03)\end{array}$ & $\begin{array}{c}0.92 \\
(0.80-1.04)\end{array}$ \\
\hline 142 & 50.4 & & 0.97 & & $(0.77-1.17)$ & $\begin{array}{c}1.02 \\
(0.80-1.20)\end{array}$ & & & 9398 & 51.3 & & 1.01 & $(0.97-1.05)$ & $\begin{array}{c}1.01 \\
(0.97-1.05)\end{array}$ & & & & & & & & \\
\hline $\begin{array}{l}{ }^{\dagger} \text { Preterm } \\
\text { birth }\end{array}$ & 2297 & 5.2 & 420 & 5.0 & $\begin{array}{c}0.97 \\
(0.87-1.07)\end{array}$ & $\begin{array}{c}1.00 \\
(0.90-1.10)\end{array}$ & 446 & 5.1 & $\begin{array}{c}0.98 \\
(0.88-1.08)\end{array}$ & $\begin{array}{c}0.99 \\
(0.89-1.09)\end{array}$ & 55 & 6.1 & $\begin{array}{c}1.20 \\
(0.91-1.49)\end{array}$ & $\begin{array}{c}1.11 \\
(0.83-1.39)\end{array}$ & 15 & 5.3 & $\begin{array}{c}1.02 \\
(0.60-1.40)\end{array}$ & $\begin{array}{c}0.98 \\
(0.58-1.38)\end{array}$ & 936 & 5.1 & $\begin{array}{c}0.99 \\
(0.91-1.07)\end{array}$ & $\begin{array}{c}1.01 \\
(0.92-1.09)\end{array}$ \\
\hline $\begin{array}{l}{ }^{\dagger} \text { Very } \\
\text { preterm } \\
\text { birth }\end{array}$ & 292 & 0.7 & 37 & 0.4 & $\begin{array}{c}0.70 \\
(0.48-0.92)\end{array}$ & $\begin{array}{c}0.54 \\
(0.01-1.07)\end{array}$ & 60 & 0.7 & $\begin{array}{c}1.03 \\
(0.78-1.18)\end{array}$ & $\begin{array}{c}0.94 \\
(0.45-1.33)\end{array}$ & 8 & 0.9 & $\begin{array}{c}1.36 \\
(0.67-2.05)\end{array}$ & $\begin{array}{c}0.90 \\
(0.12-1.68)\end{array}$ & 3 & 1.1 & $\begin{array}{c}1.62 \\
(0.51-2.73)\end{array}$ & $\begin{array}{c}1.23 \\
(0.39-2.07)\end{array}$ & 108 & 0.6 & $\begin{array}{c}0.90 \\
(0.72-1.08)\end{array}$ & $\begin{array}{c}0.99 \\
(0.78-1.10)\end{array}$ \\
\hline $\begin{array}{l}{ }^{\dagger} \text { Small-for- } \\
\text { gestational } \\
\text { age }\end{array}$ & 4231 & 9.6 & 769 & 9.8 & $\begin{array}{c}0.96 \\
(0.89-1.03)\end{array}$ & $\begin{array}{c}1.01 \\
(0.93-1.09)\end{array}$ & 787 & 9.0 & $\begin{array}{c}0.93 \\
(0.86-1.00)\end{array}$ & $\begin{array}{c}1.02 \\
(0.93-1.11)\end{array}$ & & 110 & 12.3 & $\begin{array}{c}1.32 \\
(1.09-1.55)\end{array}$ & & 1.33 & $(1.07-1.59)$ & 24 & 8.5 & 0.88 & $(0.58-1.18)$ & $\begin{array}{c}0.84 \\
(0.55-1.13)\end{array}$ \\
\hline 1690 & 9.3 & & 0.97 & & $(0.91-1.03)$ & $\begin{array}{c}1.03 \\
(0.97-1.09)\end{array}$ & & & & & & & & & & & & & & & & \\
\hline $\begin{array}{l}\text { Apgar }<7 \\
\text { at } 5 \text { min }\end{array}$ & 632 & 1.4 & 109 & 1.3 & $\begin{array}{c}0.92 \\
(0.75-1.09)\end{array}$ & $\begin{array}{c}0.94 \\
(0.76-1.12)\end{array}$ & 117 & 1.3 & $\begin{array}{c}0.93 \\
(0.77-1.09)\end{array}$ & $\begin{array}{c}0.97 \\
(0.80-1.14)\end{array}$ & 10 & 1.1 & $\begin{array}{c}0.78 \\
(0.42-1.14)\end{array}$ & $\begin{array}{c}0.70 \\
(0.36-1.04)\end{array}$ & 3 & 1.1 & $\begin{array}{c}0.76 \\
(0.24-1.28)\end{array}$ & $\begin{array}{c}0.73 \\
(0.23-1.23)\end{array}$ & 239 & 1.2 & $\begin{array}{c}0.92 \\
(0.80-1.04)\end{array}$ & $\begin{array}{c}0.94 \\
(0.80-1.08)\end{array}$ \\
\hline $\begin{array}{l}\text { ŁAll } \\
\text { congenital } \\
\text { anomalies }\end{array}$ & 2262 & 4.9 & 455 & 5.2 & $\begin{array}{c}1.08 \\
(0.97-1.19)\end{array}$ & $\begin{array}{c}1.09 \\
(1.00-1.18)\end{array}$ & 427 & 4.7 & $\begin{array}{c}0.95 \\
(0.86-1.04)\end{array}$ & $\begin{array}{c}0.98 \\
(0.88-1.08)\end{array}$ & 47 & 5.0 & $\begin{array}{c}1.04 \\
(0.77-1.31)\end{array}$ & $\begin{array}{c}1.08 \\
(0.80-1.36)\end{array}$ & 11 & 3.8 & $\begin{array}{c}0.77 \\
(0.42-1.12)\end{array}$ & $\begin{array}{c}0.65 \\
(0.30-1.00)\end{array}$ & 940 & 4.9 & $\begin{array}{c}1.01 \\
(0.94-1.08)\end{array}$ & $\begin{array}{c}1.03 \\
(0.95-1.11)\end{array}$ \\
\hline $\begin{array}{l}\mathrm{t}^{\dagger} \text { All } \\
\text { congenital } \\
\text { anomalies }\end{array}$ & 2158 & 4.8 & 437 & 5.2 & $\begin{array}{c}1.08 \\
(0.99-1.17)\end{array}$ & $\begin{array}{c}1.10 \\
(1.00-1.20)\end{array}$ & 417 & 4.7 & $\begin{array}{c}0.97 \\
(0.87-1.07)\end{array}$ & $\begin{array}{c}1.00 \\
(0.89-1.11)\end{array}$ & 45 & 5.0 & $\begin{array}{c}1.03 \\
(0.76-1.30)\end{array}$ & $\begin{array}{c}1.08 \\
(0.79-1.37)\end{array}$ & 11 & 3.8 & $\begin{array}{c}0.79 \\
(0.43-1.15)\end{array}$ & $\begin{array}{c}0.68 \\
(0.35-1.01)\end{array}$ & 910 & 5.0 & $\begin{array}{c}1.03 \\
(0.95-1.11)\end{array}$ & $\begin{array}{c}1.04 \\
(0.96-1.12)\end{array}$ \\
\hline $\begin{array}{l}\text { +q"Major" } \\
\text { congenital } \\
\text { anomalies }\end{array}$ & 1839 & 4.1 & 374 & 4.5 & $\begin{array}{c}1.09 \\
(0.97-1.21)\end{array}$ & $\begin{array}{c}1.11 \\
(1.00-1.22)\end{array}$ & 337 & 3.8 & $\begin{array}{c}0.92 \\
(0.82-1.02)\end{array}$ & $\begin{array}{c}0.95 \\
(0.84-1.06)\end{array}$ & 37 & 4.1 & $\begin{array}{c}1.00 \\
(0.71-1.29)\end{array}$ & $\begin{array}{c}1.04 \\
(0.74-1.34)\end{array}$ & 11 & 3.8 & $\begin{array}{c}0.94 \\
(0.51-1.37)\end{array}$ & $\begin{array}{c}0.79 \\
(0.40-1.18)\end{array}$ & 759 & 4.1 & $\begin{array}{c}1.01 \\
(0.92-1.10)\end{array}$ & $\begin{array}{c}1.03 \\
(0.94-1.12)\end{array}$ \\
\hline $\begin{array}{l}\text { 9Multiple } \\
\text { major } \\
\text { congenital } \\
\text { anomalies }\end{array}$ & 455 & 1.0 & 89 & 1.0 & $\begin{array}{c}1.04 \\
(0.83-1.25)\end{array}$ & $\begin{array}{c}1.09 \\
(0.85-1.33)\end{array}$ & 81 & 0.9 & $\begin{array}{c}0.90 \\
(0.71-1.09)\end{array}$ & $\begin{array}{c}1.00 \\
(0.77-1.23)\end{array}$ & 11 & 1.2 & $\begin{array}{c}1.20 \\
(0.66-1.74)\end{array}$ & $\begin{array}{c}1.28 \\
(0.70-1.86)\end{array}$ & 4 & 1.4 & $\begin{array}{c}1.42 \\
(0.60-2.24)\end{array}$ & $\begin{array}{c}1.34 \\
(0.49-2.19)\end{array}$ & 185 & 1.0 & $\begin{array}{c}0.99 \\
(0.83-1.15)\end{array}$ & $\begin{array}{c}1.06 \\
(0.90-1.22)\end{array}$ \\
\hline
\end{tabular}

${ }^{1}$ Chemist's assistants, physiotherapists, dental nurses, laboratory technicians, doctors, auxiliary nurses and nurses

${ }^{2}$ Child-minders, specialist teachers, teachers of common school, upper secondary school teachers, care assistant, educationalists and educationalist assistants.

${ }^{3}$ Kitchen assistant and butcher.

${ }^{4}$ Skiled farm worker, slaughter house worker.

${ }^{¥}$ Adjusted for maternal age, parity, history of miscarriage, socio-occupational status, pre-pregnancy body mass index, smoking habit and alcohol consumption

(as well as for sex of child for preterm and very preterm birth).

EAmong live births.

${ }^{\dagger}$ Among singletons.

IEUROCAT Classification. 
Table 4 Odds Ratios for pregnancy outcomes according to infections during pregnancy in exposed occupational groups

\begin{tabular}{|c|c|c|c|c|c|c|c|c|c|c|c|c|c|c|c|c|c|c|}
\hline & \multicolumn{2}{|c|}{ Miscarriage* } & \multicolumn{2}{|c|}{ Preterm birth } & \multicolumn{2}{|c|}{$\begin{array}{l}\text { Very preterm } \\
\text { birth }\end{array}$} & \multicolumn{2}{|c|}{$\begin{array}{c}\text { Small for } \\
\text { gestational age }\end{array}$} & \multicolumn{2}{|c|}{$\begin{array}{c}\text { Apgar }<7 \text { at } 5 \\
\text { min }\end{array}$} & \multicolumn{2}{|c|}{$\begin{array}{l}\text { All congenital } \\
\text { anomalies } \\
\text { (among live } \\
\text { births) }\end{array}$} & \multicolumn{2}{|c|}{$\begin{array}{l}\text { All congenital } \\
\text { anomalies } \\
\text { (among live } \\
\text { singletons) }\end{array}$} & \multicolumn{2}{|c|}{$\begin{array}{l}\text { Major congenital } \\
\text { anomalies } \\
\text { (among live } \\
\text { singletons) }\end{array}$} & \multicolumn{2}{|c|}{$\begin{array}{l}\text { Multiple major } \\
\text { congenital } \\
\text { anomalies }\end{array}$} \\
\hline & $\begin{array}{c}\text { ORc } \\
\text { (IC95\%) }\end{array}$ & $\begin{array}{l}\mathrm{ORa}^{*} \\
\text { (IC95\%) }\end{array}$ & $\begin{array}{c}\text { ORc } \\
\text { (IC95\%) }\end{array}$ & $\begin{array}{l}\mathrm{ORa}^{*} \\
\text { (IC95\%) }\end{array}$ & $\begin{array}{c}\text { ORc } \\
\text { (IC95\%) }\end{array}$ & $\begin{array}{l}\text { ORa }^{*} \\
\text { (IC95\%) }\end{array}$ & $\begin{array}{c}\text { ORc } \\
\text { (IC95\%) }\end{array}$ & $\begin{array}{l}\mathrm{ORa}^{*} \\
\text { (IC95\%) }\end{array}$ & $\begin{array}{c}\text { ORc } \\
\text { (IC95\%) }\end{array}$ & $\begin{array}{l}\mathrm{ORa}^{*} \\
\text { (IC95\%) }\end{array}$ & $\begin{array}{c}\text { ORc } \\
\text { (IC95\%) }\end{array}$ & $\begin{array}{l}\text { ORa }^{*} \\
\text { (IC95\%) }\end{array}$ & $\begin{array}{c}\text { ORc } \\
\text { (IC95\%) }\end{array}$ & $\begin{array}{l}\mathrm{ORa}^{*} \\
\text { (IC95\%) }\end{array}$ & $\begin{array}{c}\text { ORc } \\
\text { (IC95\%) }\end{array}$ & $\begin{array}{c}\text { ORa } \\
\text { (IC95\%) }\end{array}$ & $\begin{array}{c}\text { ORc } \\
\text { (IC95\%) }\end{array}$ & $\begin{array}{l}\mathrm{ORa}^{*} \\
\text { (IC95\%) }\end{array}$ \\
\hline \multicolumn{19}{|c|}{ Work with patients } \\
\hline \multicolumn{19}{|c|}{ Infection } \\
\hline No & 1 (ref.) & 1 (ref.) & 1 (ref.) & 1 (ref.) & 1 (ref.) & 1 (ref.) & 1 (ref.) & 1 (ref.) & 1 (ref.) & 1 (ref.) & 1 (ref.) & 1 (ref.) & 1 (ref.) & 1 (ref.) & 1 (ref.) & 1 (ref.) & 1 (ref.) & 1 (ref.) \\
\hline Yes & $\begin{array}{l}1.29 \\
(0.60- \\
1.98)\end{array}$ & $\begin{array}{c}0.35 \\
(0.01- \\
1.00)\end{array}$ & $\begin{array}{l}1.15 \\
(0.92- \\
1.38)\end{array}$ & $\begin{array}{l}1.17 \\
(0.93- \\
1.41)\end{array}$ & $\begin{array}{l}1.17 \\
(0.46- \\
1.88)\end{array}$ & $\begin{array}{l}1.03 \\
(0.40- \\
1.66)\end{array}$ & $\begin{array}{l}1.04 \\
(0.89- \\
1.19)\end{array}$ & $\begin{array}{l}1.06 \\
(0.90- \\
1.22)\end{array}$ & $\begin{array}{c}0.92 \\
(0.60- \\
1.24)\end{array}$ & $\begin{array}{c}0.99(0.64- \\
1.46)\end{array}$ & $\begin{array}{c}0.98(0.80- \\
1.16)\end{array}$ & $\begin{array}{l}1.03 \\
(0.84- \\
1.23)\end{array}$ & $\begin{array}{c}0.99(0.80- \\
1.18)\end{array}$ & $\begin{array}{l}1.04 \\
(0.84- \\
1.24)\end{array}$ & $\begin{array}{c}1.01(0.81- \\
1.21)\end{array}$ & $\begin{array}{l}1.07 \\
(0.85- \\
1.29)\end{array}$ & $\begin{array}{l}1.16 \\
(0.73- \\
1.59)\end{array}$ & $\begin{array}{l}1.29 \\
(0.80- \\
1.78)\end{array}$ \\
\hline \multicolumn{19}{|c|}{ Work with children } \\
\hline \multicolumn{19}{|c|}{ Infection } \\
\hline No & 1 (ref.) & 1 (ref.) & 1 (ref.) & 1 (ref.) & 1 (ref.) & 1 (ref.) & 1 (ref.) & 1 (ref.) & 1 (ref.) & 1 (ref.) & 1 (ref.) & 1 (ref.) & 1 (ref.) & 1 (ref.) & 1 (ref.) & 1 (ref.) & 1 (ref.) & 1 (ref.) \\
\hline Yes & $\begin{array}{l}1.53 \\
(0.74- \\
2.32)\end{array}$ & $\begin{array}{l}1.49 \\
(0.34- \\
2.64)\end{array}$ & $\begin{array}{c}0.95 \\
(0.77- \\
1.13)\end{array}$ & $\begin{array}{c}0.92 \\
(0.73- \\
1.11)\end{array}$ & $\begin{array}{c}0.86 \\
(0.38- \\
1.34)\end{array}$ & $\begin{array}{l}0.72 \\
(0.30- \\
1.14)\end{array}$ & $\begin{array}{l}1.04 \\
(0.89- \\
1.19)\end{array}$ & $\begin{array}{l}1.00 \\
(0.84- \\
1.16)\end{array}$ & $\begin{array}{c}0.92 \\
(0.62- \\
1.22)\end{array}$ & $\begin{array}{c}0.95(0.63- \\
1.27)\end{array}$ & $\begin{array}{c}0.94(0.76- \\
1.12)\end{array}$ & $\begin{array}{c}0.95 \\
(0.76- \\
1.14)\end{array}$ & $\begin{array}{c}0.93(0.75- \\
1.11)\end{array}$ & $\begin{array}{c}0.94 \\
(0.75- \\
1.13)\end{array}$ & $\begin{array}{c}0.99(0.78- \\
1.20)\end{array}$ & $\begin{array}{l}1.00 \\
(0.78- \\
1.22)\end{array}$ & $\begin{array}{l}1.09 \\
(0.67- \\
1.47)\end{array}$ & $\begin{array}{l}1.12 \\
(0.68- \\
1.56)\end{array}$ \\
\hline \multicolumn{19}{|c|}{ Work with food } \\
\hline \multicolumn{19}{|c|}{ Infection } \\
\hline No & 1 (ref.) & 1 (ref.) & 1 (ref.) & 1 (ref.) & 1 (ref.) & 1 (ref.) & 1 (ref.) & 1 (ref.) & 1 (ref.) & 1 (ref.) & 1 (ref.) & 1 (ref.) & 1 (ref.) & 1 (ref.) & 1 (ref.) & 1 (ref.) & 1 (ref.) & 1 (ref.) \\
\hline Yes & $\begin{array}{l}1.19 \\
(0.20- \\
2.18)\end{array}$ & - & $\begin{array}{c}0.86 \\
(0.46- \\
1.26)\end{array}$ & $\begin{array}{c}0.84 \\
(0.43- \\
1.25)\end{array}$ & $\begin{array}{l}1.05 \\
(0.07- \\
2.03)\end{array}$ & $\begin{array}{c}0.86 \\
(0.03- \\
1.69)\end{array}$ & $\begin{array}{l}1.35 \\
(0.87- \\
1.83)\end{array}$ & $\begin{array}{l}1.30 \\
(0.81- \\
1.79)\end{array}$ & $\begin{array}{c}0.79 \\
(0.18- \\
1.40)\end{array}$ & $\begin{array}{l}1.17(0.22- \\
2.12)\end{array}$ & $\begin{array}{c}1.01(0.55- \\
1.47)\end{array}$ & $\begin{array}{c}1.04 \\
(0.55- \\
1.53)\end{array}$ & $\begin{array}{c}1.06(0.57- \\
1.55)\end{array}$ & $\begin{array}{l}1.11 \\
(0.57- \\
1.65)\end{array}$ & $\begin{array}{l}1.35(0.68- \\
2.02)\end{array}$ & $\begin{array}{l}1.43 \\
(0.69- \\
2.17)\end{array}$ & $\begin{array}{l}1.27 \\
(0.39- \\
2.15)\end{array}$ & $\begin{array}{l}1.18 \\
(0.34- \\
2.02)\end{array}$ \\
\hline \multicolumn{19}{|c|}{ Work with animals } \\
\hline \multicolumn{19}{|c|}{ Infection } \\
\hline No & 1 (ref.) & 1 (ref.) & 1 (ref.) & 1 (ref.) & 1 (ref.) & 1 (ref.) & 1 (ref.) & 1 (ref.) & 1 (ref.) & 1 (ref.) & 1 (ref.) & 1 (ref.) & 1 (ref.) & 1 (ref.) & 1 (ref.) & 1 (ref.) & 1 (ref.) & 1 (ref.) \\
\hline Yes & - & - & $\begin{array}{c}0.88 \\
(0.23- \\
1.53)\end{array}$ & $\begin{array}{l}1.50 \\
(0.31- \\
2.69)\end{array}$ & - & - & $\begin{array}{l}0.87 \\
(0.33- \\
1.41)\end{array}$ & $\begin{array}{l}1.27 \\
(0.41- \\
2.13)\end{array}$ & $\begin{array}{l}1.36 \\
(0.17- \\
2.55)\end{array}$ & $\begin{array}{c}5.76(0.96- \\
10.12)\end{array}$ & $\begin{array}{l}9.31(1.15- \\
17.47)\end{array}$ & $\begin{array}{l}2.93 \\
(1.24- \\
4.62)\end{array}$ & $\begin{array}{l}9.41(1.16- \\
17.66)\end{array}$ & $\begin{array}{l}2.80 \\
(1.23- \\
4.37)\end{array}$ & $\begin{array}{l}9.41(1.16- \\
17.66)\end{array}$ & $\begin{array}{l}2.80 \\
(1.23- \\
4.37)\end{array}$ & $\begin{array}{l}3.34 \\
(0.34- \\
6.68)\end{array}$ & $\begin{array}{c}0.24 \\
(0.01- \\
1.00)\end{array}$ \\
\hline \multicolumn{19}{|c|}{ All exposed groups } \\
\hline \multicolumn{19}{|c|}{ Infection } \\
\hline No & 1 (ref.) & 1 (ref.) & 1 (ref.) & 1 (ref.) & 1 (ref.) & 1 (ref.) & 1 (ref.) & 1 (ref.) & 1 (ref.) & 1 (ref.) & 1 (ref.) & 1 (ref.) & 1 (ref.) & 1 (ref.) & 1 (ref.) & 1 (ref.) & 1 (ref.) & 1 (ref.) \\
\hline Yes & $\begin{array}{l}1.34 \\
(0.54- \\
2.24)\end{array}$ & $\begin{array}{c}0.92 \\
(0.32- \\
1.52)\end{array}$ & $\begin{array}{l}1.03 \\
(0.89- \\
1.17)\end{array}$ & $\begin{array}{l}1.02 \\
(0.88- \\
1.14)\end{array}$ & $\begin{array}{c}0.99 \\
(0.54- \\
1.44)\end{array}$ & $\begin{array}{c}0.86 \\
(0.46- \\
1.26)\end{array}$ & $\begin{array}{l}1.05 \\
(0.95- \\
1.15)\end{array}$ & $\begin{array}{c}1.04 \\
(0.93- \\
1.13)\end{array}$ & $\begin{array}{c}0.94 \\
(0.71- \\
1.17)\end{array}$ & $\begin{array}{c}1.00(0.75- \\
1.25)\end{array}$ & $\begin{array}{c}0.99(0.86- \\
1.12)\end{array}$ & $\begin{array}{c}1.01 \\
(0.87- \\
1.15)\end{array}$ & $\begin{array}{c}0.99(0.85- \\
1.15)\end{array}$ & $\begin{array}{c}1.01 \\
(0.87- \\
1.15)\end{array}$ & $\begin{array}{c}1.04(0.89- \\
1.17)\end{array}$ & $\begin{array}{c}1.08 \\
(0.92- \\
1.24)\end{array}$ & $\begin{array}{c}1.16 \\
(0.85- \\
1.47)\end{array}$ & $\begin{array}{c}1.23 \\
(0.89- \\
1.57)\end{array}$ \\
\hline
\end{tabular}

${ }^{¥}$ Adjusted for maternal age, parity, history of miscarriage, socio-occupational status, pre-pregnancy body mass index, smoking habit, alcohol consumption (as well as for sex of child for preterm and very preterm birth and occupation in the group of "all exposed".

MEUROCAT Classification.

*In this variable the sample size is small because many of the women who lost the baby along his pregnancy did not answer the second interview. 
occupations who reported infections were at higher risk of having children with complications.

\section{Conclusions}

Despite preventive strategies, working in specific jobs during pregnancy may impose a higher risk of infections, and working in some of these occupations may impose a slightly higher risk of CAs in their offspring. Most other reproductive failures were not increased in these occupations.

\section{List of abbreviations}

BMI: body mass index; CAs: congenital anomalies; CMV: Cytomegalovirus; DNBC: Danish National Birth Cohort; OR: odds ratios; 95\%CI: 95\% confidence interval.

\section{Acknowledgements}

The Danish National Research Foundation has established the Danish Epidemiology Science Centre that initiated and created the Danish National Birth Cohort (DNBC). The Cohort is a result of a major grant from this Foundation. The Danish Work Environment Fund financed the $\mathrm{ICOH} 88$ coding of occupations in DNBC and supported this study (MINERVA 2008). The authors wishes to thanks Professor Jorn Olsen, Department of Epidemiology, School of Public Health, UCLA, Los Angeles, USA, for check the manuscript, Inge Eisensee, Danish Epidemiology Science Centre, University of Aarhus, Denmark, for data set preparation and Hanne Tulinius, Research Secretary of Department of Occupational and Environmental Medicine, Copenhagen University Hospital, Denmark, for her help in the preparation of the manuscript.

We acknowledge to Conselleria de Educación (Dirección General de Política Científica), Generalitat Valenciana, Spain, for support to this study. We acknowledge collaboration from participating patients, control persons, hospitals, and data providers such as the mothers because without them, this study would not have been possible.

\section{Author details}

${ }^{1}$ Unit of Public Health and Environmental Care, Department of Preventive Medicine, University of Valencia, Valencia, Spain. ${ }^{2}$ CIBER Epidemiology and Public Health (CIBERESP), Spain. ${ }^{3}$ Center for Public Health Research (CSISP), Valencia, Spain. ${ }^{4}$ The Danish Epidemiology Science Centre. University of Aarhus, Aarhus, Denmark. ${ }^{5}$ Centre for National Clinical Databases South, Dept. of Research and HTA, Odense University Hospital, Denmark. ${ }^{6}$ Department of Occupational Medicine, Aarhus University Hospital, Aarhus, Denmark. ${ }^{7}$ Department of Epidemiology, School of Public Health, UCLA, Los Angeles, USA.

\section{Authors' contributions}

MMMSV organized the study and wrote the manuscript. JO and had the original idea for the work, provided expert advice on study organization and contributed to the writing of the manuscript. JPB contributed to the study design and contributed to the writing of the manuscript. LK, JLZ and EAN contributed by interpreting the results and helped to write the manuscript. MMMSV, ALLG and NGC did the data analysis and contributed to the writing of the paper. All the authors read and approved the final manuscript.

\section{Competing interests}

The authors declare that they have no competing interests.

Received: 23 July 2010 Accepted: 15 November 2010 Published: 15 November 2010

\section{References}

1. Driscoll T, Takala J, Steenland K, Corvalan C, Fingerhut M: Review of estimates of the global burden of injury and illness due to occupational exposures. Am J Ind Med 2005, 48:491-502.
2. Sever LE: Congenital malformations related to occupational reproductive hazards (review). Occup Med 1994, 9:471-494.

3. Rasmussen SA, Erickson JD, Reef SE, Ross DS: Teratology: From science to birth defects prevention. Birth Defects Res A Clin Mol Teratol 2009, 85:82-92.

4. Jamieson DJ, Theiler RN, Rasmussen SA: Emerging infections and pregnancy. Emerg Infect Dis 2006, 12:1638-1643.

5. Valenti WM: Infection control and the pregnant health care workers. Nurs Clin North Am 1993, 28:673-686.

6. McGrath BJ: Identying health and safety risk for childcare workers. AAOHN J 2007, 55:321-325.

7. Hofmann F: Biological hazards in the work environment. Bun Ges Ges 2008, 51:313-321.

8. Triche EW, Hossain N: Environmental factors implicated in the causation of adverse pregnancy outcome. Semin Perinatol 2007, 31:240-242.

9. Jamieson DJ, Honein MA, Rasmusesen SA, Williams JL, Swerdlow DL, Biggerstaff MS, Lindstrom S, Louie JK, Christ CM, Bohm SR, Fonseca VP, Ritger KA, Kuhles DJ, Eggers P, Bruce H, Davidson HA, Lutterloh E, Harris ML, Burke C, Cocoros N, Finelli L, MacFarlane KF, Shu B, Olsen SJ, Novel Influenza A (H1N1) Pregnancy Working Group: H1N1 2009 influenza virus infection during pregnancy in the USA. Lancet 2009, 374:451-458.

10. Acs N, Bánhidy F, Horváth-Puhó E, Czeizel AE: Population-based casecontrol study of the common cold during pregnancy and congenital abnormalities. Eur J Epidemiol 2006, 21:65-75.

11. Al-Khan A, Caligiuri A, Apuzzio J: Parvovirus B-19 infection during pregnancy. Infect Dis Obstet Gynecol 2003, 11:175-179.

12. Boggess KA, Moss K, Madianos P, Murtha AP, Beck J, Offenbacher S: Fetal immune response to oral pathogens and risk of preterm birth. Am J Obstet Gynecol 2005, 193:1121-1126.

13. Resnik R: Intrauterine growth restriction. Obstet Gynecol 2002, 99:490-496.

14. Bale JF Jr: Congenital infections. Neurol Clin 2002, 20:1039-1060.

15. Mets MB: Eye manifestations of intrauterine infections. Ophthalmol Clin North Am 2001, 14:521-531.

16. Miller JR: Persistent infection by poliovirus: experimental studies. Adv Neurol 1982, 36:311-316.

17. Olsen J, Melbye M, Olsen SF, Sørensen TI, Aaby P, Andersen AM, Taxbøl D, Hansen KD, Juhl M, Schow TB, Sørensen HT, Andresen J, Mortensen EL, Olesen AW, Søndergaard C: The Danish National Birth Cohort-its background, structure and aim. Scand J Public Health 2001, 29:300-307.

18. Nohr EA, Rrydenberg M, Henriksen TB, Olsen J: Does low participation in cohort studies induce bias? Epidemiology 2006, 17:413-418

19. Zhu J, Hjollund NH, Andersen AM, Olsen J: Shift work, job stress, and late fetal loss: the National Birth Cohort in Denmark. J Occup Environ Med 2004, 46:1144-1149.

20. Disco-88: Statistics Denmark's Standard Classification of Occupation. Copenhagen: Danmarks Statistik 1996.

21. Weyers $\mathrm{H}$ : The standardized evaluation of newborn infants using the Apgar score. Dtsch Schwesternztg 1966, 7:320-322.

22. EUROCAT Guide 1.2 Instructions for the registration of CongenitalAnomales. UK EUROCAT Central Registry, January 2002. [http://www.eurocat.ulster.ac.uk].

23. Morales-Suarez-Varela MM, Nohr EA, Llopis-Gonzalez A, Andersen AM, Olsen J: Socio-occupational status and congenital anomalies. Eur J Public Health 2009, 19:161-167.

24. Suárez-Varela MM, Kaerlev L, Zhu JL, Bonde JP, Nøhr EA, Llopis-González A Olsen J: Hospital work and pregnancy outcomes: a study in the Danish National Birth Cohort. Int J Occup Environ Health 15:402-409.

25. Sharbaugh RJ: The risk of occupational exposure and infection with infectious diseases: Part 3. Home Care Provid 1999, 4:58-59.

26. Gaytant M, Steegers E, Semmekrot BA, Merkus HM, Galama JM: Congenital cytomegalovirus infection: review of the epidemiology and outcome. Obst Gynecol Surv 2002, 57:245-256

27. Stelma FF, Smismans A, Goossens VJ, Bruggeman CA, Hoebe CJ: Occupational risk of human cytomegalovirus and parvovirus B19 infection in female day care personnel in the Netherlands; a study based on seroprevalence. Eur J Clin Microbiol Infect Dis 2009, 28:393-397.

28. Hilebrant L, Harmanli $O$, Whiteman V, Khandelwal M: Urinary tract infection in pregnant women with bacterial vaginosis. Am J Obstet Gynecol 2002, 186:916-917.

29. Su SB, Wang JN, Lu CW, Guo HR: Reducing urinary tract infections among female clean room workers. J Womens Health (Larchmt) 2006, 15:870-876. 
30. Hofmann F: Biological hazards in the work environment. Bundesgesundheitsblatt Gesundheitsforschung Gesundheitsschutz 2008, 51:313-321.

31. Stelma FF, Smismans A, Goossens VJ, Bruggeman CA, Hoebe CJ: Occupational risk of human cytomegalovirus and parvovirus B19 infection in female day 01care personnel in the Netherlands; a study based on seroprevalence. Eur J Clin Mocribiol Infect Dis 2009, 28:393-397.

32. Zhu JL, Knudsen LE, Andersen AM, Hjollund NH, Olsen J: Laboratory work and pregnancy outcomes: a study within the National Birth Cohort in Denmark. Occup Environ Med 2006, 63:53-58.

33. Yasuda T, Aoki K, Matsumoto A, Mayurama K, Hyodo-Taguchi Y, Fushiki S, Ishikawa Y: Radiation-induced brain cell death can be observed in living Medaka embryos. J Radiat Res (Tokio) 2006, 47:295-303.

34. Walusiak J, Wagrowska-Koski E, Palczynski C: Health effects of occupational exposure to cytostatics in medicinal personnel in the light of compulsory prophylactics: a cross-sectional study. Med Pre 2003, 54:229-236.

35. Zhu JL, Hjollund NH, Boggild H, Olsen J: Shift work and subfecundity: a causal link or an artefact? Occup Environ Med 2003, 60:12-17.

36. Lawson CC, Whelan EA, Hibert EN, Grajewski B, Spiegelman D, RichEdwards JW: Occupational factors and risk of preterm birth in nurses. Am J Obst Gynecol 2009, 200, 51.e1-58.

37. Kaerlev L, Jacobsen LB, Bonde JP, Olsen J: Long-term sick leave and its risk factors during pregnancy among Danish hospital employees. Scand J Public Health 2004, 32:111-117.

doi:10.1186/1476-069X-9-70

Cite this article as: Morales-Suárez-Varela et al:. Risk of infection and adverse outcomes among pregnant working women in selected occupational groups: A study in the Danish National Birth Cohort.

Environmental Health 2010 9:70.

\section{Submit your next manuscript to BioMed Central and take full advantage of:}

- Convenient online submission

- Thorough peer review

- No space constraints or color figure charges

- Immediate publication on acceptance

- Inclusion in PubMed, CAS, Scopus and Google Scholar

- Research which is freely available for redistribution

Submit your manuscript at www.biomedcentral.com/submit 